\title{
LETTER
}

\section{Distinction between induction and maintenance dosing in continuous renal replacement therapy}

\author{
Graeme MacLaren* \\ See related review by Prowle et al., http://ccforum.com/content/15/2/207
}

In their excellent review of dosing continuous renal replacement therapy (CRRT), Dr Prowle and colleagues concluded that patients should be prescribed 20 to $25 \mathrm{ml} / \mathrm{kg} / \mathrm{h}$ [1]. However, by averaging CRRT dose over time, studies in this area obfuscate the benefits of appropriately higher dose therapy early in the course of illness, potentially misguiding clinicians into blindly adopting a 'one-size-fits-all' approach and consequently prescribing inadequate doses in life-threatening emergencies. To take a crude example, it would be inappropriate to prescribe $20 \mathrm{ml} / \mathrm{kg} / \mathrm{h}$ CRRT in a patient with serum potassium $9 \mathrm{mmol} / \mathrm{L}$. Rather, the highest possible dose of CRRT should be initially prescribed to maximize solute clearance. This depends on the maximum circuit flow permitted by the access catheter, which in turn determines the maximum dose, assuming that the countercurrent flow to blood flow ratio should be $<0.3$ with diffusive CRRT, or a filtration fraction with convective therapy $<0.2[2]$. As the potassium level falls, the dose can be lowered to more conventional levels.

Parallels could be drawn to general anaesthesia, where induction and maintenance are two distinct phases with different requirements. CRRT prescription could similarly be conceptualized as 'induction', where life-threatening abnormalities are corrected quickly with high-dose therapy, then 'maintenance' where solute clearance is achieved with more temperate doses (for example, 20 to $25 \mathrm{ml} / \mathrm{kg} / \mathrm{h}$ ) to avoid complications such as hypophosphataemia.

It seems unlikely that this issue will be the subject of prospective research. Yet the principle that faster correction of life-threatening abnormalities leads to better patient outcomes seems both practical and intuitive.

\section{Authors' response}

John R Prowle and Rinaldo Bellomo

We thank Dr MacLaren for his comments. We agree that such 'induction' therapy is mandatory in situations of severe hyperkalemia $(>8.0 \mathrm{mmol})$, especially when ongoing potassium release is taking place (for example, rhabdomyolysis). Although such reasoning and therapeutic adjustment seemed obvious to us, we agree that making them explicit is important.

We would also like to emphasize several important aspects of adjustments to unique cases. First, avoidance of often ineffective and delayed interventions in critically ill patients with severe acute kidney injury [3] and early CRRT as applied in the RENAL (Randomised Evaluation of Normal versus Augmented Level of Replacement

*Correspondence: gmaclaren@iinet.net.au

Cardiothoracic ICU, National University Hospital, 5 Lower Kent Ridge Road, Singapore 119074
Therapy) trial [4-6] are key to preventing such lifethreatening events in the first place. In this regard, in the RENAL trial, only 6 out of $1,454(0.4 \%)$ patients with baseline electrolyte values had a potassium level $>8 \mathrm{mmol} / \mathrm{L}$. Second, faster solute removal can be achieved by the application of modified dialytic techniques that deliver full equilibration between plasma flow and dialysate flow and much greater solute clearance [7]. Third, the concept of 'induction' applies to volume control as well: in a patient with pulmonary edema or receiving large amounts of blood products, the intensity of volume removal should be adjusted accordingly. Finally, however, also a word a caution: in the RENAL trial, three patients suffered from disequilibrium syndrome because of too rapid solute removal. In patients with very high concentrations of azotemic markers, overly intensive solute removal can be detrimental. Similarly, in patients with marked hyper- or hyponatremia, rapid normalization can be dangerous and induce cerebral edema or demyelination. 
In the end, for RRT as well as every other medical therapy, there is no substitute for a trained, educated, committed, diligent and thoughtful physician.

\section{Abbreviations}

CRRT, continuous renal replacement therapy; RENAL, Randomised Evaluation of Normal versus Augmented Level of Replacement Therapy.

\section{Competing interests}

The author declares that he has no competing interests

Published: 26 April 2011

\section{References}

1. Prowle JR, Schneider A, Bellomo R: Clinical review: Optimal dose of continuous renal replacement therapy in acute kidney injury. Crit Care 2011, 15:207.

2. Ricci Z, Ronco C: Dose and efficiency of renal replacement therapy: Continuous renal replacement therapy versus intermittent hemodialysis versus slow extended daily dialysis. Crit Care Med 2008, 36:S229-237.
3. Bagshaw SM, Delaney A, Haase M, Ghali WA, Bellomo R: Loop diuretics in the management of acute renal failure: a systematic review and metaanalysis. Crit Care Resusc 2007, 9:60-68.

4. The RENAL Study Investigators: The RENAL (Randomised Evaluation of Normal vs. Augmented Level of Replacement Therapy) study: statistical analysis plan. Crit Care Resusc 2009, 11:58-66.

5. RENAL Study Investigators: Renal replacement therapy for acute kidney injury in Australian and New Zealand intensive care units: a practice survey. Crit Care Resusc 2008, 10:225-230.

6. The RENAL Replacement Therapy Study Investigators: Intensity of continuous renal replacement therapy in critically ill patients. New Engl J Med 2009, 361:1627-1638

7. Bellomo R, Baldwin I, Fealy N: Prolonged intermittent renal replacement therapy in the intensive care unit. Crit Care Resusc 2002, 4:281-290.

doi:10.1186/cc10137

Cite this article as: MacLaren G: Distinction between induction and maintenance dosing in continuous renal replacement therapy. Critical Care 2011, 15:419. 